PACIFIC JOURNAL OF MATHEMATICS

Vol. 186, No. 1, 1998

\title{
BOCHNER'S TECHNIQUE ON LORENTZIAN MANIFOLDS AND INFINITESIMAL CONFORMAL SYMMETRIES
}

\author{
Alfonso Romero and Miguel Sánchez
}

\begin{abstract}
Bochner's technique is applied to the study of timelike vector fields on a Lorentzian manifold. A Lorentzian Bochner integral formula is obtained; as a consequence, compact Ricciflat Lorentzian manifolds admitting a timelike conformal vector field are classified. Some obstructions to the existence of timelike conformal vector fields and other conformal symmetries are also given.
\end{abstract}

\section{Introduction.}

In a previous article [9] the authors introduced an integral inequality on compact Lorentzian manifolds and applied it to Killing vector fields. As a remarkable fact, this inequality was obtained by using Bochner's technique, for the first time in the Lorentzian case, to our knowledge. The main obstruction to apply Bochner's technique to Lorentzian manifolds seems to be the following (see $[\mathbf{1 0}]$ for a pedagogical approach). Recall that there are two slightly different points of view about classical Bochner's technique [15]; the first one uses an integral formula obtained as a consequence of the divergence theorem; the second takes into account the ellipticity of the Laplacian associated to a Riemannian metric, especially the classical maximum principle. As was shown in [14], this last point of view allow to refine, in some directions, the results obtained from the first approach. For a Lorentzian metric, the D'Alambertian is formally defined as the Laplacian of a Riemannian metric, but now this operator is not elliptic; this fact leads to the absence, in the Lorentzian case, of the well-known properties of the Riemannian one (note, for instance, that on a compact Lorentzian manifold, "harmonic" functions are not necessarily constant). Thus, it seems impossible to translate this especially useful second approach to the Lorentzian case. On the other hand, classical Bochner's results made assumptions on curvature which are misleading or may have more than one translation for a Lorentzian metric (for instance, trivially, a "negative semidefinite Ricci tensor" in the case of a Lorentzian metric with constant sectional curvature $c$ implies $c=0$ ). Even more, there are some additional tools in the Riemannian case for the geometric interpretations of the results, which do not hold for a Lorentzian metric 
(for example, the isometry group of a compact Lorentzian manifold is not necessarily compact [3], see also [11]). Taking into account these difficulties, our startpoint in [9] was to introduce Bochner's technique regarding timelike Killing vector fields. The purpose of this article is to introduce Bochner's technique for timelike vector fields (not necessarily Killing) in a more general setting. As a particular consequence, several classification and nonexistence results obtained in [9] for the Killing case are generalized to the conformal one.

This paper is laid out as follows. In Section 2 an integral formula for timelike vector fields is obtained, Theorem 2.1. This formula can be seen as a version of classical Bochner's formula [1] adapted to the difficulties of the Lorentzian case. In our formula, timelike vector fields are normalized (reference frames) to obtain algebraically manageable terms. An integral inequality involving an arbitrary reference frame is then obtained, Corollary 2.2, and discussed, Remark 2.3. In Section 3 these results are applied to study certain infinitesimal conformal symmetries. The application of Bochner's technique to conformal vector fields in the Riemannian case was first carried out by Yano [16]. The study of conformal vector fields in Lorentzian geometry is more subtle than in the Riemannian case, and has been developed mainly under assumptions of interest in Physics (see, for example [2], [4]). Of course, stronger results has been proved for the special case of Killing vector fields, which appear as a useful tool to get classification results in some areas of Lorentzian Geometry. In particular, standard space forms (those with a time-orientable double covering admitting a timelike Killing vector field) have been studied in [6], [7]. The authors do not know previous results in this direction for the conformal case. Our main result is obtained in Theorem 3.6; as an interesting consequence, we prove a uniqueness result about compact Ricci-flat Lorentzian manifolds admitting a timelike conformal vector field, Corollary 3.9. Moreover, nonexistence results for timelike conformal vector fields, under certain Ricci curvature assumptions, are obtained in Corollary 3.8 and Corollary 3.10.

Even though our objective in Section 3 is just to obtain strictly mathematical results (in the spirit, for example, of [6], [9], and references therein), it leads to consider spatially conformally stationary reference frames (see Definition 3.3). These vector fields generalize the rigid reference frames, which are well-known in General Relativity [12], and have been recently studied in [5]. Lorentzian manifolds admitting them are more general than those admitting a timelike conformal vector field, Example 3.5. At the beginning of Section 3, we obtain some of their properties which are relevant in our approach (compare with [5, Definition 3.1 and Remark 3.2]). 


\section{Lorentzian Bochner Formula.}

Let $(M, g)$ be a $n$-dimensional Lorentzian manifold, that is, a (connected, smooth) manifold $M$ endowed with a non-degenerate metric $g$ with in$\operatorname{dex}(+, \ldots,+,-)$. As usual, the causal character of a tangent vector $v \in$ $T_{p} M, p \in M$ is timelike (resp. null, spacelike) if $g(v, v)<0$ (resp. $g(v, v)=$ $0, g(v, v)>0)$. For any vector field, $X \in \mathcal{X}(M)$ consider the tensor field $A_{X}$ of type $(1,1)$ given by $A_{X}(v)=-\nabla_{v} X$, where $\nabla$ denotes the Levi-Civita connection of $g$. Classical Bochner's formula asserts, for compact $M$ :

$$
\int_{M}\left\{\operatorname{Ric}(X, X)+\operatorname{trace}\left(A_{X}^{2}\right)-\left(\operatorname{trace} A_{X}\right)^{2}\right\} d v=0 .
$$

Observe that this formula is true in the Lorentzian case as well as in the Riemannian one; in fact, it is obtained just taking into account that the integral of the divergence of the vector field $W=-A_{X}(X)-(\operatorname{div} X) X$ vanishes. Now, put

$$
\delta_{X}=\operatorname{trace}\left(A_{X}^{2}\right)-\left(\operatorname{trace} A_{X}\right)^{2} .
$$

If $\delta_{X}$ has a definite sign, Bochner's formula (1) yields a strong link between $X$ and the sign of the Ricci curvature $\operatorname{Ric}(X, X)$. In fact, the more classical applications in the Riemannian case are obtained by applying (1) on certain vector fields (Killing, harmonic, conformal) for which $\delta_{X}$ is signed; then, it follows that $\operatorname{Ric}(X, X)$ must have opposite sign to $\delta_{X}$ at some points. For the Lorentzian case none of the analogous conditions on $X$ imply that $\delta_{X}$ is signed, even when $X$ has a definite causal character (timelike, null or spacelike).

Now, assume that $Z \in \mathcal{X}(M)$ is a reference frame, that is, $g(Z, Z)=-1$. Then $A_{Z}$ can be restricted to the subspace $Z^{\perp}$ orthogonal to $Z$, and it is straightforward to check that the corresponding restriction $A_{Z}^{\prime}$ also satisfies:

$$
\delta_{Z}=\operatorname{trace}\left(A_{Z}^{\prime 2}\right)-\left(\operatorname{trace} A_{Z}^{\prime}\right)^{2} .
$$

Put $A_{Z}^{\prime}=S_{Z}^{\prime}+H_{Z}^{\prime}$ where $S_{Z}^{\prime}$ (resp. $H_{Z}^{\prime}$ ) is, at any point $p$, a self-adjoint (resp. skew-adjoint) operator on $Z^{\perp}$ at $p$. Then (3) can be written as:

$$
\delta_{Z}=-s_{Z}^{\prime}+\operatorname{trace}\left(H_{Z}^{\prime 2}\right),
$$

with $s_{Z}^{\prime}=-\operatorname{trace}\left(S_{Z}^{\prime 2}\right)+\left(\operatorname{trace} S_{Z}^{\prime}\right)^{2}$. From (4), (2) and (1) the following integral formula is obtained.

Theorem 2.1 (Lorentzian Bochner formula). Let $(M, g)$ be a compact Lorentzian manifold and let $Z$ be a reference frame on $(M, g)$. Then

$$
\int_{M}\left\{\operatorname{Ric}(Z, Z)-s_{Z}^{\prime}+\operatorname{trace}\left(H_{Z}^{\prime 2}\right)\right\} d v=0
$$


When $n=2$ then $s_{Z}^{\prime} \equiv 0, H_{Z}^{\prime} \equiv 0$, and integral formula (5) is just GaussBonnet theorem. On the other hand, note that $\operatorname{trace}\left({H_{Z}^{\prime}}^{2}\right) \leq 0$ with equality at some point $p$ if and only if $H_{Z}^{\prime}=0$ at $p$. So, as a consequence of Theorem 2.1 we have:

Corollary 2.2. Let $(M, g)$ be a compact Lorentzian manifold and let $Z$ be a reference frame on $(M, g)$. If $s_{Z}^{\prime} \geq 0$ then

$$
\int_{M} \operatorname{Ric}(Z, Z) d v \geq 0
$$

and the equality holds if and only if $s_{Z}^{\prime} \equiv 0$ and $H_{Z}^{\prime} \equiv 0$.

Remark 2.3. Let $\lambda_{1}(p), \cdots, \lambda_{n-1}(p)$ be the eigenvalues of the operator $S_{Z}^{\prime}$ at the point $p$. Then $s_{Z}^{\prime}(p)=\sum_{i \neq j} \lambda_{i}(p) \lambda_{j}(p)$, that is, $s_{Z}^{\prime}$ is, up to a sign, the coefficient of $t^{n-3}$ in the characteristic polynomial of $S_{Z}^{\prime}$ at $p$. As a consequence, if all the $\lambda_{i}(p)$ have equal sign at each point $p$ then $s_{Z}^{\prime} \geq 0$. Moreover, in this case, $s_{Z}^{\prime}(p)=0$ if and only if the $\lambda_{i}(p)$ are all zero but, at most, one.

\section{Application to infinitesimal conformal symmetries.}

We begin showing two complementary results. The first one characterizes the tensor field $S_{Z}^{\prime}$; the second one will be useful to apply Lorentzian Bochner formula, in particular, to any timelike vector field (not necessarily unit). We denote by $\mathcal{L}_{X}$ the Lie derivative with respect to $X \in \mathcal{X}(M)$.

Lemma 3.1. For each reference frame $Z$ consider the decomposition $A_{Z}=$ $S_{Z}+H_{Z}$ where $S_{Z}\left(\right.$ resp. $\left.H_{Z}\right)$ is a self-adjoint (resp. skew-adjoint) operator with respect to $g$. Then $T_{Z}:=-(1 / 2) \mathcal{L}_{Z} g$ is the 2 -covariant tensor field g-equivalent to $S_{Z}$ (that is, $g\left(X, S_{Z}(Y)\right)=T_{Z}(X, Y)$, for all $X, Y \in \mathcal{X}(M)$ ).

In particular, the restriction $T_{Z}^{\prime}$ of $T_{Z}$ to $Z^{\perp}$ is g-equivalent to the restricted operator $S_{Z}^{\prime}$.

Proof. Note that

$$
2 T_{Z}(X, Y)=g\left(X, S_{Z}(Y)\right)+g\left(S_{Z}(X), Y\right)
$$

from which the result directly follows.

The following lemma is also a consequence of (7).

Lemma 3.2. Let $X$ be a timelike vector field on $(M, g)$ and let $f \in C^{\infty}(M)$. If $T_{X}=-(1 / 2) \mathcal{L}_{X} g$ then

$$
2 f T_{X}(U, V)=-\left(\mathcal{L}_{f X} g\right)(U, V)
$$

for all $U, V$ in $X^{\perp}$. 
Recall that a vector field $C \in \mathcal{X}(M)$ is called conformal if $\mathcal{L}_{C} g=2 \mathrm{~kg}$, where $k: M \rightarrow \mathbb{R}$, and $C$ is Killing if it is conformal with $k \equiv 0$; a Lorentzian manifold is called stationary if it admits a timelike Killing vector field. Following [5] we introduce the next concept.

Definition 3.3. Let $(M, g)$ be a Lorentzian manifold. A reference frame $Z$ is spatially conformally stationary $(S C S$ ) provided that

$$
\left(\mathcal{L}_{Z} g\right)(U, V)=2 k g(U, V)
$$

for all $U, V \in \mathcal{X}(M)$ orthogonal to $Z$, where $k: M \rightarrow \mathbb{R}$. Moreover, if $k \equiv 0$ then $Z$ is called spatially stationary $(S S)$.

SS reference frames are also called rigid in the literature $[\mathbf{1 2}$, p. 56]. In General Relativity these vector fields model sets of observers which see a constant metric in the spatial part for them. SCS reference frames model observers which see an expansion or compression along their proper time. The existence of conformal symmetries is a quite general and useful assumption to study Einstein equations.

The natural behaviour of these vector fields under conformal changes of metric is stated in the following result.

Proposition 3.4. Let $(M, g)$ be a Lorentzian manifold and put $g^{*}=$ $\exp (2 u) g$, where $u \in C^{\infty}(M)$.

(i) If a vector field $X$ is conformal for $g$ then it is conformal for $g^{*}$. Moreover, if $g^{*}(X, X)$ is a nonzero constant then $X$ is Killing for $g^{*}$.

(ii) If $X$ is a SCS reference frame in $(M, g)$ then $X^{*}=\left(-g^{*}(X, X)\right)^{-1 / 2} \cdot X$ is a $S C S$ reference frame in $\left(M, g^{*}\right)$.

Proof. Note that

$$
\mathcal{L}_{X} g^{*}=2 X(u) g^{*}+\exp (2 u) \mathcal{L}_{X} g
$$

which yields the first assertion in (i). For the second one, note that any (local) flow $\Phi_{t}$ of $X$ is a conformal transformation for each $t$, and the corresponding conformal factor can be obtained as $g^{*}\left(\Phi_{t *}(X), \Phi_{t *}(X)\right) / g^{*}(X, X)$. Thus, assertion (ii) follows from formula (8) and Lemma 3.2.

Example 3.5. (1) From Lemma 3.2, if a timelike vector field $C$ is conformal (resp. Killing) the normalized vector field $Z=(-g(C, C))^{-1 / 2} \cdot C$ is a SCS (resp. SS) reference frame. Not all the SCS reference frames can be obtained in such a way, as the next example shows in particular. 
Consider the Lorentzian metric $g=d x^{2}-G(x+y) d y^{2}$ on $\mathbb{R}^{2}$, where: (i) $G$ is periodic, and, thus, $g$ can be induced on a torus $T^{2}$, (ii) $G>0$ (i.e. $g$ is Lorentz), and (iii) $G$ is not constant. A direct computation shows that $\partial / \partial x-\partial / \partial y$ yields a Killing vector field $K$ on $T^{2}$. This is the unique Killing vector field on $T^{2}$ (up to a constant) because, in the opposite case, $g$ would be flat [11], in contradiction with (iii). It is straightforward to check that the reference frame $Z$ on $T^{2}$ induced from $G(x+y)^{-1 / 2} \cdot \partial / \partial y$ is SS. $Z$ is not the normalized of a Killing vector field because of the uniqueness of $K$. Moreover, $Z$ is neither the normalized of any timelike conformal (nonKilling) vector field $C$. In fact, if $Z=(-g(C, C))^{-1 / 2} \cdot C$, then $\mathcal{L}_{C} g=2 \sigma g$, with $\sigma \not \equiv 0$ and, $\mathcal{L}_{Z} g=2(-g(C, C))^{-1 / 2} \cdot \sigma g$ on $Z^{\perp}$; thus, $\mathcal{L}_{Z} g \neq 0$ on $Z^{\perp}$, a contradiction. Even more, for any conformal metric $g^{*}$, the vector field $Z^{*}=\left(-g^{*}(Z, Z)\right)^{-1 / 2} \cdot Z$ is SCS (in fact, every reference frame on a Lorentz surface is SCS) but it is not the normalized of any conformal vector field.

(Note also that if $G$ reaches the value 1 , then $g$ is geodesically incomplete [11], which is an obstruction to the existence of a timelike conformal vector field on $T^{2}$, see [8]. On the other hand, if (ii) is replaced by: (ii') $G<0$, then, analogous examples are suggested for a Riemannian metric.)

(2) Denote by $\pi: S^{2 n+1} \longrightarrow \mathbb{C} P^{n}$ the usual Hopf fibration from an odddimensional (unitary) sphere $S^{2 n+1}$ to a complex projective space $\mathbb{C} P^{n}$. For each $u \in C^{\infty}\left(S^{2 n+1}\right)$ put $g_{u}=\exp (2 u) \pi^{*} g_{F}-\omega \otimes \omega$, where $g_{F}$ is the Fubini metric on $\mathbb{C} P^{n}$ and $\omega$ is the 1-form naturally obtained from the usual connection on this fibre bundle. Thus, each $g_{u}$ is a Lorentzian metric on $S^{2 n+1}$ with the same SCS reference frame $Z$ given by $w \longrightarrow \sqrt{-1} . w$, for all $w \in S^{2 n+1} \subset \mathbb{C}^{n+1}$. Note that, in this example, the distribution $Z^{\perp}$ is not integrable.

Next, we will apply Corollary 2.2 to a SCS reference frame $Z$. Note that such a $Z$ has $s_{Z}^{\prime}=(n-1)(n-2) k^{2}$, and hence $s_{Z}^{\prime} \geq 0$. Moreover, if $n \geq 3$ then the equality holds if and only if $T_{Z}^{\prime} \equiv 0$. Thus we have:

Theorem 3.6. Let $(M, g)$ be a compact Lorentzian manifold with dimension $n \geq 3$. If $(M, g)$ admits a SCS reference frame $Z$ then

$$
\int_{M} \operatorname{Ric}(Z, Z) d v \geq 0
$$

and the equality holds if and only if $A_{Z}^{\prime} \equiv 0$.

Remark 3.7. From Proposition 3.4, the existence of a SCS reference frame is a conformal invariant. Note that the property $\operatorname{Ric}(Z, Z)>0$ is not conformal invariant; in fact, if this inequality holds at a point $p$ then a conformal metric $g^{*}$ can be constructed such that its Ricci tensor satisfies 
$\operatorname{Ric}^{*}(Z, Z)<0$ at a neighborhood of $p$. In the compact case, $\int_{M} \operatorname{Ric}(Z, Z) d v$ is not necessarily equal to the corresponding integral $\int_{M} \operatorname{Ric}^{*}\left(Z^{*}, Z^{*}\right) d v^{*}$, where $Z^{*}=\left(-g^{*}(Z, Z)\right)^{-1 / 2} \cdot Z$, but both integrals must be non-negative. This fact can be seen as a conformal invariant property of the conclusion in Theorem 3.6.

Corollary 3.8. A compact Lorentzian manifold with $\operatorname{Ric}(X, X)<0$ for all timelike vector field $X$ admits no SCS reference frame.

In particular, a compact Einstein Lorentzian manifold with $\mathrm{Ric}=c g$ admitting a SCS reference frame must satisfy $c \leq 0$.

For the case $c=0$, the following strong result holds. Note that it is necessary to assume the existence of a timelike conformal vector field, not only a SCS reference frame.

Corollary 3.9. Let $(M, g)$ be an n-dimensional compact Ricci-flat Lorentzian manifold admitting a timelike conformal vector field $C$. Then $C$ is parallel, the first Betti number of $M$ is not zero, and the Levi-Civita connection of $g$ coincides with the Levi-Civita connection of a Riemannian metric.

Moreover, $(M, g)$ is isometric to a flat Lorentzian n-torus, up to a (finite) covering if one of the following three conditions holds:

(1) $(M, g)$ is homogeneous,

(2) $(M, g)$ is flat,

(3) $n \leq 4$.

Proof. The case $n=2$ is trivial, so assume $n \geq 3$. By Theorem 3.6 with $Z=(-g(C, C))^{-1 / 2} \cdot C$, we have $A_{Z}^{\prime} \equiv 0$. A direct computation using Lemma 3.2 shows that $C$ is in fact Killing; moreover, by using (1) we have $A_{Z} \equiv 0$, that is, $C$ is parallel. Then, the result follows from [9, Theorem 3.2].

Finally, we have the following nonexistence result.

Corollary 3.10. A simply connected compact Lorentzian manifold $(M, g)$ with $\operatorname{Ric}(X, X) \leq 0$ for all timelike vector field $X$ admits no timelike conformal vector fields.

Proof. The same argument as in the previous result shows that such a vector field would be parallel. Moreover $(M, g)$ is geodesically complete from the result in [8]. Now de Rham-Wu decomposition theorem [13] can be claimed, and $(M, g)$ is isometric to a metric product $\mathbb{R} \times M^{\prime}$, where $M^{\prime}$ is a Riemannian manifold. But this contradits the compactness assumption. 


\section{References}

[1] S. Bochner, Vector fields and Ricci curvature, Bull. Amer. Math. Soc., 52 (1946), 776-797.

[2] A.A. Coley and B.O.J. Tupper, Special conformal Killing vector space-times and symmetry inheritance, J. Math. Phys., 30 (1989), 2616-2625.

[3] G. D'Ambra, Isometry groups of Lorentz manifolds, Invent. Math., 92 (1988), 555565.

[4] D. Earley, J. Isenberg, J. Marsden and V. Moncrief, Homothetic and conformal symmetries of solutions to Einstein's equations, Commun. Math. Phys., 106 (1986), 137-158.

[5] E. García-Río and D.N. Kupeli, Singularity versus splitting theorems for stably causal spacetimes, Annals Glob. Anal. Geom., 14 (1996), 301-312.

[6] Y. Kamishima, Completeness of Lorentz manifolds of constant curvature admitting Killing vector fields, J. Differential Geom., 37 (1993), 569-601.

[7] R. Kulkarni and F. Raymond, 3-dimensional Lorentz space-forms and Seifert fiber spaces, J. Differential Geom., 21 (1985), 231-268.

[8] A. Romero and M. Sánchez, Completeness of Lorentz manifolds admitting a timelike conformal Killing vector field, Proc. Amer. Math. Soc., 123 (1995), 2831-2833.

[9] _ An integral inequality on compact Lorentz manifolds and its applications, Bull. London Math. Soc., 28 (1996), 509-513.

[10] , An introduction to Bochner's technique on Lorentzian manifolds, Proc. of the V Fall Worshop: Differential Geometry and its applications to Mathematical Physics, Jaca, Spain, 1996.

[11] M. Sánchez, Structure of Lorentzian tori with a Killing vector field, Trans. Amer. Math. Soc., 349(3) (1997), 1063-1080.

[12] R. Sachs and H. Wu, General Relativity for Mathematicians, Graduate Texts in Math., 48, Springer, New York, 1977.

[13] H. Wu, On the Rham decomposition theorem, Illinois J. Math., 8 (1964), 291-311.

[14] A remark on the Bochner technique in differential geometry, Proc. Amer. Math. Soc., 78 (1980), 403-408.

[15] - The Bochner Technique, Proc. of the 1980 Beijing symposium on Differential Geometry and Differential Equations, II, 929-1071.

[16] K. Yano, On harmonic and Killing vector fields, Ann. Math., 55 (1952), 38-45.

Received April 24, 1997 and revised September 29, 1997. The authors were partially supported by a DGICYT Grant PB-94-0796.

FACUltad DE Ciencias

Universidad DE GRANADA

18071-Granada, SPAIN

E-mail address: aromero@goliat.ugr.es

sanchezm@goliat.ugr.es 